Review Article

\title{
Biomarkers as Predictors of Recurrence following Curative Resection for Pancreatic Ductal Adenocarcinoma: A Review
}

\author{
Sylvester N. Osayi, Mark Bloomston, Carl M. Schmidt, \\ E. Christopher Ellison, and Peter Muscarella
}

Department of Surgery and Center for Minimally Invasive Surgery, The Ohio State University Wexner Medical Center, Columbus, $\mathrm{OH} 43210$, USA

Correspondence should be addressed to Sylvester N. Osayi; sylvester.osayi@osumc.edu

Received 10 January 2014; Revised 2 June 2014; Accepted 3 June 2014; Published 24 June 2014

Academic Editor: Steven N. Hochwald

Copyright (C) 2014 Sylvester N. Osayi et al. This is an open access article distributed under the Creative Commons Attribution License, which permits unrestricted use, distribution, and reproduction in any medium, provided the original work is properly cited.

Pancreatic ductal adenocarcinoma (PDA) is the fourth most common cancer causing death in the United States. Early tumor recurrence is an important contributor to the dismal prognosis. The availability of an accurate prognostic biomarker for predicting disease recurrence following curative resection will be beneficial for patient care. Most of the currently studied biomarkers remain in the investigational phase, with CA 19-9 being the only biomarker currently approved by the FDA. Herein, we review the utility of CA 19-9 and other investigational cellular, gene, and molecular tumor markers for predicting PDA recurrence following curative surgical resection.

\section{Introduction}

Pancreatic ductal adenocarcinoma (PDA) remains a lethal disease. It is the fourth most common cause of cancer-related death in the United States with an estimated incidence of 45,220 new cases and 38,460 deaths in 2013. The 5-year survival continues to be approximately $5 \%$ [1]. Surgical resection, though not without significant morbidity and mortality, provides the only chance for obtaining cure. Nonetheless, only about $20 \%$ of cases are localized and resectable at the time of presentation, and the 5-year survival following "curative" resection remains less than 25\% [2].

Tumor recurrence is one of the main contributors to poor survival after curative resection of PDA. Many patients develop local recurrence and distant metastasis even following resection for early staged disease $[3,4]$. Consequently, the ability to predict subgroups of patients with a high likelihood of early recurrence may be beneficial in the appropriate management of the disease. This information might be useful for selecting the timing and appropriateness of surgical intervention and neoadjuvant and adjuvant systemic chemotherapies. In addition, the considerable morbidity and mortality of major pancreatic resections could be avoided in patients who are unlikely to benefit as determined by preoperative assessment using accurate biomarkers.

The ability to successfully manage PDA is limited by the lack of accurate disease biomarkers. Tumor markers can be employed as tools for screening, diagnosis, prognosis, and surveillance. Though numerous diagnostic and prognostic PDA biomarkers have been proposed, most of them are still in the investigational phase. A comprehensive review of the literature in 2009 showed that more than 2300 papers have been published on over 2500 overexpressed genes that could all serve as biomarkers for PDA [5]. These numbers are expected to increase with ongoing findings of newly identified overexpressed genes. Despite the considerable amount of work that has been done in this area, CA 19-9 remains the only widely used biomarker in clinical practice today. Our interest here is in the prognostic utility of biomarkers as predictors of PDA recurrence following curative resection. This review aims to provide an overview of the use of CA 19-9 and other promising prognostic biomarkers (Table 1) to predict PDA recurrence following surgical resection. 
TABLE 1: Biomarkers evaluated for predicting recurrence following resection of pancreatic ductal adenocarcinoma.

\begin{tabular}{l}
\hline Carbohydrate antigen 19-9 (CA 19-9) \\
Carcinoembryonic antigen (CEA) \\
Cellular biomarkers \\
Circulating tumor cells (CTCs) \\
Neutrophil-lymphocyte ratio (NLR) \\
Gene biomarkers \\
P16/CDKN2A, TP53, and SMAD4/DPC4 \\
Metastin \\
Phosphatase and tensin (PTEN) \\
Molecular biomarkers \\
CX chemokine receptor 4 (CXCR4) \\
Cathepsin B \\
Vascular endothelial growth factor (VEGF) \\
MicroRNAs (miRNAs)
\end{tabular}

\section{Methods}

2.1. Literature Search. A systematic review of the PUBMED database was performed according to the Preferred Reporting Items for Systematic Review and Meta-Analyses (PRISMA) guidelines [6]. The database was searched for relevant articles published on biomarkers for predicting pancreatic cancer recurrence following curative resection. To identify relevant studies, the terms "pancreatic," "cancer," "adenocarcinoma," "recurrence", and "biomarker" were used. The search was limited to publications from 1990 to present. An initial search resulted in 223 studies. Articles in languages other than English were excluded. Next, the titles and abstracts were screened for irrelevant topics, which were excluded. Full text appraisal was performed on original studies that focused on evaluating prognostic biomarkers in patients who underwent curative resection and that addressed survival and disease recurrence as one of their endpoints. In addition, the references of the full text articles were checked for relevant publications. These studies were included in the final review. Figure 1 shows the search strategy and the number of included original studies. A summary of the 15 original studies included in this review is shown in Table 2.

\section{Discussion}

3.1. Carbohydrate Antigen 19-9 (CA 19-9). CA 19-9 is the most widely studied PDA tumor marker and the only FDA approved biomarker for PDA [22]. It was first described in 1979 using a monoclonal antibody (MAb) directed against the colon cancer cell line SW1116 [23]. It has since been most commonly utilized in the setting of pancreatic cancer $[24,25]$. CA 19-9 is derived from an abnormal pathway during production of its normal equivalent, disialyl Lewis ${ }^{\mathrm{a}}$. The antigenic determinant of the MAb to CA19-9 is related to a sialylated Lewis ${ }^{\mathrm{a}}$ blood group and targets either $\mathrm{Le}^{\mathrm{a}+}$ or $\mathrm{Le}^{\mathrm{b}+}$ forms depending on the presence of specific fucosylation patterns (Figure 2). As a result, the utility of this serum biomarker is limited in patients who are Lewis blood type negative $\left(\mathrm{Le}^{\mathrm{a}-\mathrm{b}-}\right)$. These individuals fail to express high level of CA19-9, even in the presence of high tumor burden. They are estimated to include $7-10 \%$ of the population $[26,27]$. Another limitation of CA $19-9$ is that it can be falsely elevated in the presence of biliary obstruction $[26,28]$. CA 19-9 has an estimated sensitivity of $71-81 \%$ and specificity of $83-90 \%$ for the diagnosis of PDA at a cut-off level of $37 \mathrm{U} / \mathrm{mL}[24,29]$.

In addition to the diagnostic utility of CA 19-9 in PDA, some studies have specifically assessed its ability to predict PDA recurrence following curative resection. Sugiura and colleagues evaluated preoperative CA 19-9 as a predictor of early recurrence (defined as relapse $<6$ months after resection) in 154 patients using a cut-off value of $100 \mathrm{U} / \mathrm{mL}$ [7]. Among 73 patients with CA $19-9$ value $\geq 100 \mathrm{U} / \mathrm{mL}, 39$ (53\%) had early recurrence compared to 9 of 81 patients $(11 \%)$ with CA $19-9$ value $<100 \mathrm{U} / \mathrm{mL}(P<0.001)$. CA $19-9$ value $\geq$ $100 \mathrm{U} / \mathrm{mL}$ had an odds ratio of 11.2 in predicting early recurrence. Patients with CA 19-9 value $<100 \mathrm{U} / \mathrm{mL}$ had 3and 5 -year survival rates of $47.3 \%$ and $40.1 \%$ versus $21.2 \%$ and $9.4 \%$, respectively, with a median survival time of 31 versus 16 months, compared to patients whose CA 19-9 values were $\geq 100 \mathrm{U} / \mathrm{mL}(P<0.001)$.

In light of the limitation of CA 19-9 in the presence of biliary obstruction, some investigators have devised alternative methods of interpreting CA 19-9 levels. Kang et al. postulated that an adjusted preoperative CA 19-9 level might be a better predictor of prognosis [8]. They calculated an adjusted CA 199 level by dividing the serum CA 19-9 by the total bilirubin in patients with bilirubin greater than $2 \mathrm{mg} / \mathrm{dL}$. The authors observed that patients with adjusted preoperative CA 19-9 levels $>50 \mathrm{U} / \mathrm{mL}$ had recurrence risk twice that of patients with levels $<50 \mathrm{U} / \mathrm{uL}(P=0.027)$. Parameters predictive of recurrence on univariate analysis included adjusted CA 199 level $\geq 50 \mathrm{U} / \mathrm{mL}(P=0.0049)$, peripancreatic microscopic cancer invasion $(P=0.0142)$, and lymphovascular invasion $(P=0.0038)$. Only adjusted CA $19-9$ proved predictive of 12 month disease recurrence on multivariate analysis.

In addition to assessing the prognostic value of preoperative CA 19-9, some investigators have attempted to evaluate the association between postoperative CA 19-9 level and PDA recurrence. Following curative resection, it is expected that CA 19-9 levels will return to a normal range. Postoperative normalization of CA 19-9 has been linked to improved survival [9], while failure of normalization has been correlated with metastatic disease or recurrence [30]. Tian et al. assessed the prognostic attributes of elevated postoperative CA 19-9 in patients with Lewis antigen positive blood groups [9]. The group observed local recurrence and distant metastases in 6 of $11(54.5 \%)$ patients that underwent resection. In those patients, secondary elevations in serum CA 19-9 preceded tumor recurrence by 2 to 9 months. In another study, it was observed that sustained postoperative elevation of CA19-9 level preceded clinical and radiologic detection of recurrence by 2 weeks to 5 months [31].

An investigation by Hata et al. reported a statistically significant increase in disease recurrence in patients with postoperative CA 19-9 > $37 \mathrm{U} / \mathrm{mL}$ [10]. Among this subgroup 


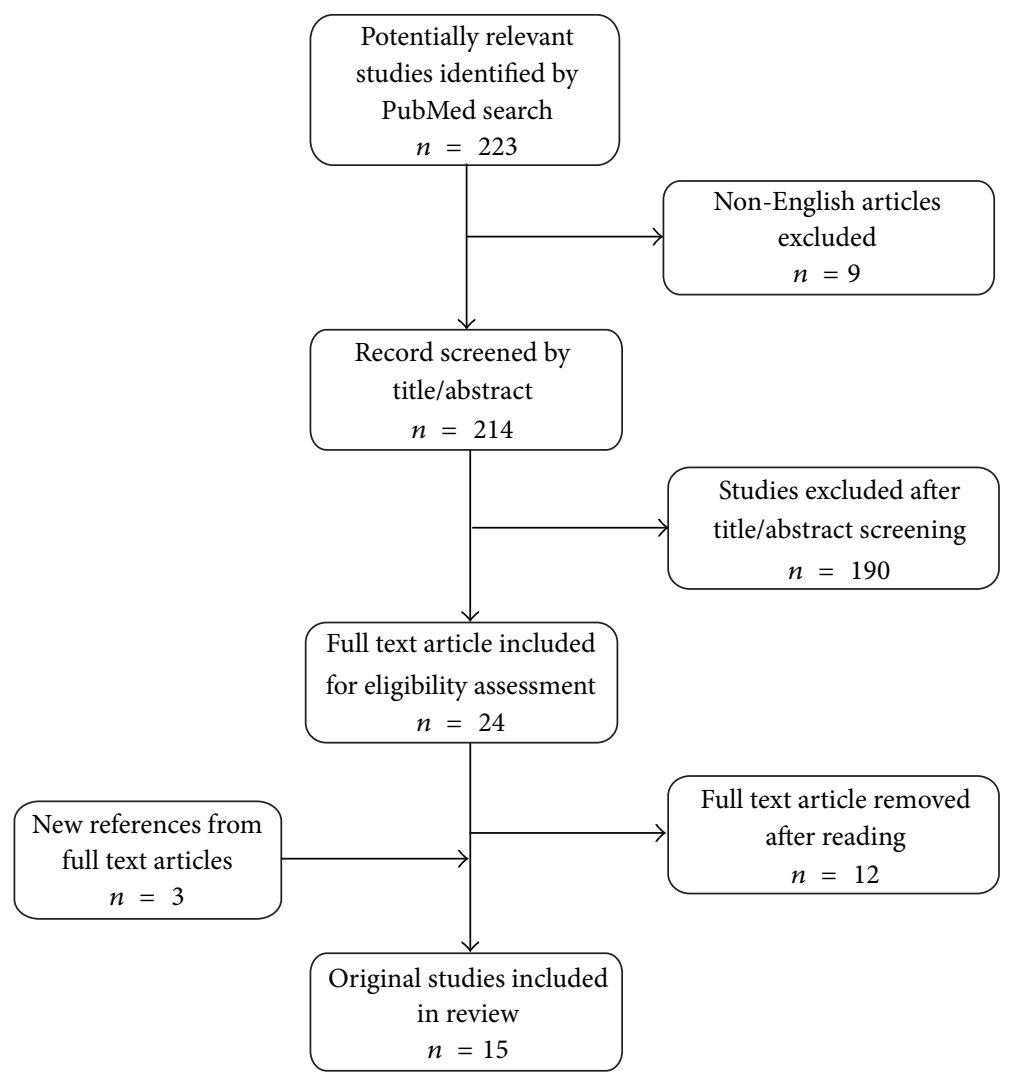

FIGURE 1: Online search strategy.

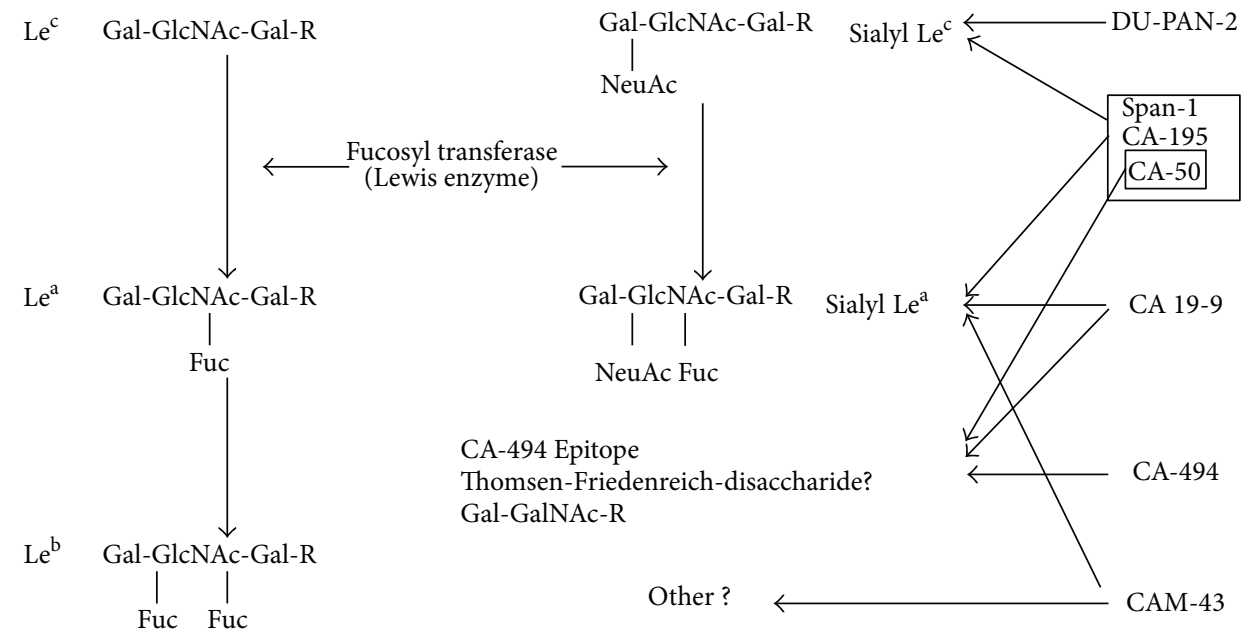

FiguRE 2: Tumor-related antigens and the carbohydrate determinants recognized by their corresponding MAbs (adapted from Muscarella II et al. [25]).

of patients, overall recurrence was $83 \%$ versus $64 \%(P=$ 0.002 ) and the rate of hepatic metastasis was $44 \%$ versus $23 \%(P<0.0001)$, significantly higher in comparison to patients with postoperative CA19-9 $\leq 37 \mathrm{U} / \mathrm{mL}$. Contrary to previously described studies $[7,8]$, this study did not show a statistically significant increase in overall recurrence with high preoperative CA 19-9 levels.
A novel interpretation of the postoperative CA 19-9 level that has been shown to be predictive of disease recurrence following surgery is CA 19-9 velocity. Hernandez et al. defined this as the rate at which CA 19-9 levels change over a 4week time frame [11]. The group measured CA 19-9 velocity in 96 patients following pancreatectomy and reported it to be a better predictor of overall survival than baseline 


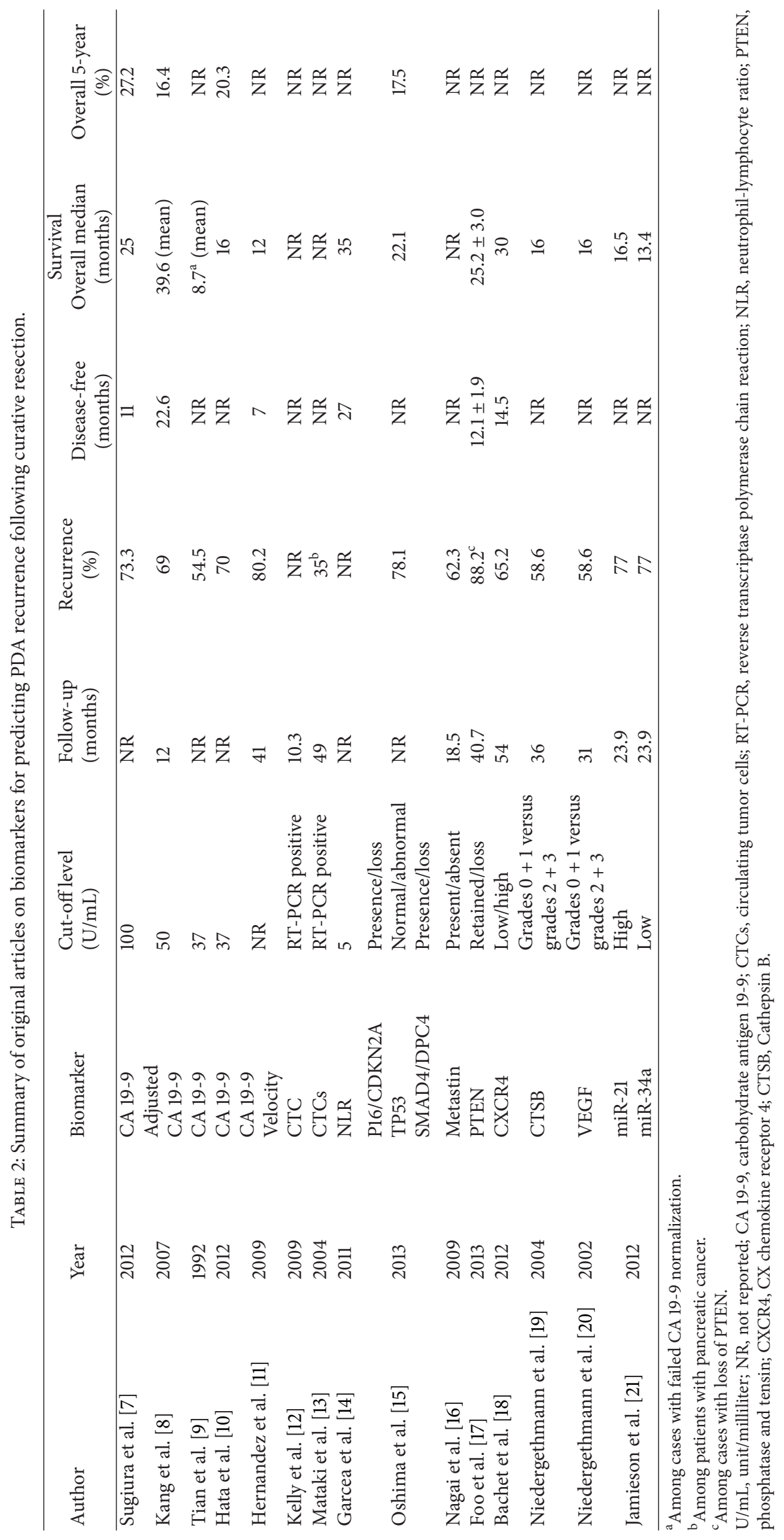


postoperative CA 19-9 $(P<0.001)$. In their study, patients with disease progression following surgery were found to have a mean velocity of $131 \mathrm{U} / \mathrm{mL} / 4$-weeks, compared to $1 \mathrm{U} / \mathrm{mL} / 4$-weeks for patients without disease progression $(P<0.001)$. The authors reported with $100 \%$ certainty that a CA 19-9 velocity of $95 \mathrm{U} / \mathrm{mL} / 4$-weeks would denote disease recurrence confirmable by radiographic imaging.

In summary, CA 19-9 is an effective marker for predicting PDA recurrence following curative resection. Preoperative CA 19-9 levels > 100 U/mL, adjusted levels > 50 U/mL, elevated postoperative CA 19-9 levels, and postoperative CA $19-9$ velocity $>95 \mathrm{U} / \mathrm{mL} / 4$-weeks have all been shown to predict disease recurrence.

3.1.1. Carcinoembryonic Antigen (CEA). Prior to the availability of CA 19-9 assays, CEA was the only serum antigen used for diagnosing PDA [25]. It has since been shown to have major drawbacks in PDA diagnosis due to its much lower sensitivities and specificities in comparison to CA 19-9 [22, 32]. It has also been shown to be inferior to CA 19-9 for monitoring disease burden and surveillance following curative resection $[33,34]$. However, Yasue et al. demonstrated the prognostic value of CEA in 90 patients who underwent PDA resection [35]. They reported a significant correlation between preoperative CEA level $>2.5 \mathrm{ng} / \mathrm{mL}$ and poor survival. A similar correlation was reported for postoperative elevation in CEA. Despite these findings, CEA remains less informative for PDA and is currently only FDA approved as a tumor marker for colon cancer.

\subsection{Cellular Biomarkers}

3.2.1. Circulating Tumor Cells (CTCs). Tumor recurrence significantly contributes to increased mortality in patients with PDA and may be driven by early metastasis. The ability to detect metastasis at an early stage might improve prognosis following surgical resection by identifying patients who are appropriate candidates for early treatment with systemic therapy. Detection of circulating tumor cell (CTC) micrometastases in peritoneal fluid and blood has been proposed as a viable method for identifying early tumor burden. The technique employs reverse transcription polymerase chain reaction (RT-PCR). RT-PCR has been shown to be a more sensitive method for detecting metastases in the peritoneal fluid during staging laparoscopy or at the time of surgical resection as compared to conventional cytology [36-38]. CEA has been utilized as the target to identify CTCs during RT-PCR [37].

Using CEA mRNA during RT-PCR, Kelly et al. evaluated the presence of micrometastases in patients undergoing $\mathrm{R} 0$ resection [12]. In their study, patients with radiologic or tissue diagnosis of PDA underwent staging laparoscopy. Normal saline was introduced into the peritoneal cavity and then aspirated and sent for cytology and RNA isolation. Amplification of CEA mRNA in less than 40 cycles was deemed a positive RT-PCR. The investigators observed that, among 62 patients who underwent R0 resection, 11 (18\%) had positive CEA-mRNA in their peritoneal washing by RT-PCR in the presence of negative cytology. These patients experienced higher rates of recurrence than those with negative RT-PCR $(P=0.003)$. RT-PCR positive status correlated with early recurrence $(P=0.01)$. It also correlated with decreased survival, though without statistical significance $(P=0.370)$.

In addition to peritoneal washing, CTCs have been investigated in the blood. Intraoperative measurement of CEA mRNA in venous blood by RT-PCR has shown prognostic value in detecting disease recurrence, especially in patients with high-stage disease $[39,40]$. Mataki et al. investigated the significance of CTCs in the blood as an early indicator of PDA recurrence following curative resection [13]. They obtained blood sample every 3 months from 53 patients who had undergone curative resection for biliary-pancreatic cancer, 20 of whom had pancreatic cancer. The samples were evaluated for CEA mRNA expression using RT-PCR. Of the 7 PDA patients diagnosed with recurrence by radiological studies, 5 of them (71\%) were blood positive for CEA mRNA. On the other hand, only 1 of the 13 PDA patients without recurrence $(7.7 \%)$ had positive CEA mRNA. CEA mRNA had a sensitivity of $75 \%$ and specificity of $94 \%$ for predicting disease recurrence in this study, much better than CEA or CA 19-9 alone. CEA mRNA positive blood predated radiological diagnosis of recurrence by about 3 months. Other studies provide further clinical relevance of blood CTCs in pancreatic cancer, including CTC enrichment and detection techniques and their clinical implications [41]. Though there is a heterogeneity of publications on the utility of CTCs in predicting PDA recurrence, the above reports provide evidence of the emerging prognostic value of CTCs.

3.2.2. Neutrophil-to-Lymphocyte Ratio (NLR). Malignancies have been suggested to incite inflammatory responses in patients. It has also been suggested that recruited inflammatory cells could negatively impact survival of several types of cancer [42, 43]. Based on this knowledge, preoperative neutrophil-to-lymphocyte ratio (NLR) has been proposed as a promising predictor of survival in patients with PDA [4446]. NLR is calculated by dividing the neutrophil count by the lymphocyte count. The sensitivity and specificity of NLR are significantly increased at levels greater than 5 [45]. Garcea et al. investigated the value of NLR in predicting prognosis following curative resection of PDA in 72 patients who underwent pancreaticoduodenectomy [14]. All patients had blood drawn preoperatively and analyzed for NLR, plateletto-lymphocyte ratio (PLR), and C-reactive protein (CRP). At follow-up of 1 to 125.8 months, perioperative NLR among patients who experienced disease recurrence was observed to be significantly higher than that of patients without recurrence (4.7 versus 3.1, $P=0.02$ ). No differences in PLR and CRP were identified between the two groups. Patients with NLR $>5$ had a median disease-free survival of 12 months, significantly less than the 52 months observed in patients with NLR $<5(P<0.001)$. The study demonstrates the prognostic utility of this easily obtainable clinical parameter. 


\subsection{Gene Biomarkers}

3.3.1. P16/CDKN2A, TP53, and SMAD4/DPC4. Several gene markers have been implicated in PDA, though four of them, termed "mountain" genes, are the most commonly mutated. The genes are KRAS, CDKN2A/p16, TP53, and SMAD4/ DPC4. KRAS is mutated in almost all PDA specimens. The individual and collective prognostic significance of these "mountain" genes in PDA have been the subject of numerous investigations, though fewer studies have specifically addressed their role in predicting disease recurrence. Oshima et al. evaluated the prognostic role of CDKN2A/p16, TP53, and SMAD4/DPC4 in 106 patients with PDA following surgical resection [15]. The patients had median and 5-year survivals of 22.1 months and $17.5 \%$, respectively. Overall, $78.1 \%$ of the patients developed disease recurrence. Loss of CDKN2A/p16 was seen in $67 \%$ of cases and was associated with lymphatic invasion $(P=0.012)$, shorter overall $(P=$ $0.029)$ and disease-free $(P=0.015)$ survivals, and distant metastases $(P=0.005)$. Abnormal TP53 was observed in $81.1 \%$ of the cases and was associated with the presence of locoregional recurrence $(P=0.020)$. SMAD4/DPC4 was absent in $54.7 \%$ of patients and this was associated with lymphatic invasion $(P=0.033)$ and shorter median overall $(P<0.001)$ and disease-free $(P<0.001)$ survivals. Noteworthy in the study was that all 6 patients with more than 5-year survival had intact SMAD4/DPC4.

Despite the above finding on SMAD4/DPC4, published reports on the utility of this gene remain contradictory. While some studies have suggested that its absence was linked with disease progression $[15,47,48]$ and distant metastases [49], more recent investigations have shown no association between this tumor marker and PDA recurrence $[18,50]$. Winter et al., in their evaluation of 127 patients who underwent resection for PDA, found no association between SMAD4 expression and PDA recurrence $(P=0.9)$ or death $(P=0.15)$ [50]. Though reports on the value of the "mountain genes" in predicting PDA recurrence are mixed in general, they remain viable prognostic biomarkers and deserve further investigation.

3.3.2. Metastin. Metastin is a gene product of the tumor suppressor gene KiSS-1 and a ligand to the G-protein-coupled receptor GPR54 [51, 52]. PDA has been shown to express lower levels of KiSS-1 mRNA and higher levels of GPR54 mRNA in comparison to normal pancreatic tissue, suggesting the role of metastin in PDA metastasis [53]. Nagai et al. investigated the prognostic role of metastin in 53 patients who underwent curative resection for PDA. Metastin was observed to be expressed in only $24.5 \%$ of tumors [16]. These metastin-positive tumors were less likely to have recurrence (38.5\% versus $70.0 \%, P=0.04$ ) and the presence of metastin significantly correlated with longer survival (hazard ratio = $2.1 ; 95 \%$ confidence interval $=1.1-4.7$; and $P=0.03$ ). Interestingly, none of the 6 patients with high plasma metastin levels in that study died during the median followup period of 18.5 months. Monitoring of metastin levels in resected tumor could offer prognostic value in predicting
PDA recurrence, though further studies are needed to bolster this claim.

3.3.3. Phosphatase and Tensin (PTEN). Phosphatase and tensin (PTEN) is a tumor suppressor gene encoded on chromosome 10q23.3. It is one of the most commonly inactivated genes in sporadic cancers and has been implicated in several human malignancies [17]. The function of PTEN can be lost by deletion, gene silencing, mutation, or dysregulation of messenger RNA by microRNAs. Multiple studies using mouse models have shown that deletion or knockout of PTEN results in metaplasia, consequently leading to PDA $[54,55]$. In about $70 \%$ of PDA, PTEN expression is either low or absent [54]. Though some studies have shown the utility of PTEN as a marker of survival in patients with hepatobiliary cancers [56], there is a paucity of studies specifically related to its usefulness for predicting PDA recurrence and survival. In one of the few related studies, Foo et al. evaluated the role of PTEN in predicting disease recurrence and survival following curative resection in 133 patients with PDA [17]. Of the $25.6 \%$ of cases with loss of PTEN, 88.2\% had local recurrence or distant metastasis versus $68.7 \%$ in cases with retained PTEN expression $(P=0.03)$. In addition, $88.2 \%$ of the cases with PTEN loss had lymph node metastasis versus $71.7 \%$ of cases with retained PTEN $(P=0.05)$. Loss of PTEN correlated with decreased overall survival (32.7 \pm 5.0 versus $19.9 \pm 3.6$ months, $P=0.03)$.

\subsection{Molecular Biomarkers}

3.4.1. CX Chemokine Receptor 4 (CXCR4). CXCR4 is a protein receptor of the CXC chemokine ligand 12 (CXCL12). It is usually overexpressed in tumor cells of epithelial origin [57]. CXCL12 is a strong chemoattractant for mature and immature hematopoietic cells. It is normally expressed in tissues like lymph node, liver and lung, and all areas susceptible to pancreatic cancer metastasis. High expression of CXCR4 in resected PDA has been associated with shorter overall survival, lymph node metastases, and liver recurrence in a small series of patients [57]. Bachet et al. investigated the prognostic value of CXCR4 in 471 patients who underwent curative resection for PDA [18]. They showed that high CXCR4 expression was significantly associated with worse outcomes in patients who did not receive an adjuvant therapy $(\mathrm{HR}=1.69 ; P=0.012)$. On multivariate analysis, high levels of CXCR4 were associated with distant recurrence $(P<$ 0.001 ) but not with locoregional recurrence. Also, CXCR4 was shown to be a strong prognostic factor when compared with other clinical and pathological factors for predicting patients' outcome following resection. This report implicates CXCR4 as a potential biomarker of distant recurrence in PDA and as an attractive therapeutic target.

3.4.2. Cathepsin B. The tendency for PDA to invade tissues and metastasize at very early stages contributes to the poor prognosis [58]. Invasion and metastasis utilize the action of proteolytic enzymes that are able to degrade components of the extracellular matrix and basement membranes $[59,60]$. 
Cathepsin B (CTSB) is a lysosomal protease that has been shown to promote local tumor invasion and distant metastasis of PDA [58, 59, 61]. Niedergethmann et al. evaluated the prognostic significance of CTSB following R0 resection in 70 patients with PDA [19]. During the 3-year followup period, $58.6 \%$ of patients had disease recurrence, with early recurrence (within 6 months of resection) occurring in $18.6 \%$ of patients. CTSB immunoreactivity in the tumor cells was noted to be $95.7 \%$. The authors observed that moderate and strong (grade 2 and 3$)$ CTSB reactivity were associated with shorter survival $(P=0.005)$ and early postoperative recurrence (within 6 months after surgery, $P=0.0001$ ) in comparison to absent or weak (grade 0 or 1 ) reactivity.

3.4.3. Vascular Endothelia Growth Factor (VEGF). Angiogenesis involves the development of new blood capillaries. It is an important factor in tumor invasion and metastases [62]. The process allows invasion of tumor cells through the newly formed, unorganized endothelial basement membrane [63]. Several growth factors work to promote angiogenesis, but the most potent of them is vascular endothelial growth factor (VEGF). VEGF has been shown to be prognostic in PDA, correlating with poor prognosis and local disease progression $[64,65]$. It has also been shown to be associated with PDA metastasis, especially with the liver [66]. While these studies have shown the utility of VEGF as a marker of disease progression and metastasis, the question of its application as an indicator of disease recurrence remains largely unanswered. Niedergethmann et al. attempted to answer this question by investigating the correlation between VEGF expression and early recurrence following curative resection [20]. They followed 70 patients who underwent curative resection for 2 years. VEGF immunoreactivity was observed in $88.8 \%$ of the patients. There was a significant correlation between VEGF immunoreactivity and cumulative survival $(P<0.05)$, and patients with high VEGF expression had significantly higher rates of tumor recurrence within 8 months of curative surgery $(P=0.003)$. The ability to utilize VEGF in predicting early PDA recurrence will potentially identify at-risk patients for new, better-targeted adjuvant treatment. Currently, there are several preclinical and clinical trials exploring the benefits of VEGF inhibition in the treatment of PDA with some promising results [67-70]. Further discussion of these trials is beyond the scope of the current review.

3.4.4. MicroRNA (miRNA). MicroRNAs (miRNAs) are noncoding molecules involved in posttranscriptional gene regulation. Their investigation encompasses a new area of study that is showing promising clinical relevance. miRNAs are able to differentiate PDA from chronic pancreatitis through their patterns of expression [71]. The list of miRNAs implicated in PDA continues to grow and includes miR-21, 34a, 196a, 211, 217, 218, 224, and 486. miRNAs have been implicated in PDA invasion and metastasis $[72,73]$ and have been reported to be predictive of disease-free and overall survival [74]. Jamieson et al. investigated the genome-wide miRNA expression using microarray analysis in 48 patients who underwent curative resection [21]. After a series of confirmatory tests, the authors identified high miR-21 and low miR-34a expressions both to predict poor overall survival. Investigations on miRNAs are a novel area of focus. Further work is needed to determine their true utility for predicting PDA recurrence following resection.

\section{Conclusion}

Although surgical resection provides the only potential for cure in patients with PDA, early disease recurrence frequently limits survival. Despite numerous investigations, the goal of identifying an ideal biomarker for predicting early disease recurrence remains incompletely realized. The ability to predict patients who are at high risk for early recurrence based on tumor biomarkers can facilitate the provision of personalized treatment and may allow for improved disease survival. Presently, CA 19-9 remains the only widely used marker for PDA and represents the best prognostic biomarker for predicting disease recurrence following curative resection. Other biomarkers, such as CEA mRNA from CTCs and preoperative tumor NLR, have shown ready clinical applicability. In addition, some gene and molecular biomarkers have been investigated with promising results. Further work is needed to identify an ideal biomarker that is both accurate and feasible for predicting PDA recurrence. We remain optimistic that such a tumor marker will become available for clinical use in the near future.

\section{Conflict of Interests}

The authors declared that there was no conflict of interests.

\section{References}

[1] R. Siegel, D. Naishadham, and A. Jemal, "Cancer statistics, 2013," CA Cancer Journal for Clinicians, vol. 63, no. 1, pp. 11-30, 2013.

[2] H. Ueno, T. Kosuge, Y. Matsuyama et al., "A randomised phase III trial comparing gemcitabine with surgery-only in patients with resected pancreatic cancer: Japanese Study Group of Adjuvant Therapy for Pancreatic Cancer," The British Journal of Cancer, vol. 101, no. 6, pp. 908-915, 2009.

[3] K. C. Conlon, D. S. Klimstra, and M. F. Brennan, "Long-term survival after curative resection for pancreatic ductal adenocarcinoma: clinicopathologic analysis of 5-year survivors," Annals of Surgery, vol. 223, no. 3, pp. 273-279, 1996.

[4] A. Richter, M. Niedergethmann, J. W. Sturm, D. Lorenz, S. Post, and M. Trede, "Long-term results of partial pancreaticoduodenectomy for ductal adenocarcinoma of the pancreatic head: 25-Year experience," World Journal of Surgery, vol. 27, no. 3, pp. 324-329, 2003.

[5] H. C. Harsha, K. Kandasamy, P. Ranganathan et al., "A compendium of potential biomarkers of pancreatic cancer," PLoS Medicine, vol. 6, no. 4, Article ID e1000046, 2009.

[6] D. Moher, A. Liberati, J. Tetzlaff, and D. G. Altman, "Preferred reporting items for systematic reviews and meta-analyses: the PRISMA statement," PLOS Medicine, vol. 6, no. 7, Article ID e1000097, 2009.

[7] T. Sugiura, K. Uesaka, H. Kanemoto et al., "Serum CA19-9 is a significant predictor among preoperative parameters for 
early recurrence after resection of pancreatic adenocarcinoma," Journal of Gastrointestinal Surgery, vol. 16, no. 5, pp. 977-985, 2012.

[8] C. M. Kang, J. Y. Kim, G. H. Choi et al., “The use of adjusted preoperative CA 19-9 to predict the recurrence of resectable pancreatic cancer," Journal of Surgical Research, vol. 140, no. 1, pp. 31-35, 2007.

[9] F. Tian, H. E. Appert, J. Myles, and J. M. Howard, "Prognostic value of serum CA 19-9 levels in pancreatic adenocarcinoma," Annals of Surgery, vol. 215, no. 4, pp. 350-355, 1992.

[10] S. Hata, Y. Sakamoto, Y. Yamamoto et al., "Prognostic impact of postoperative serum CA 19-9 levels in patients with resectable pancreatic cancer," Annals of Surgical Oncology, vol. 19, no. 2, pp. 636-641, 2012.

[11] J. M. Hernandez, S. M. Cowgill, S. Al-Saadi et al., "CA 19-9 velocity predicts disease-free survival and overall survival after pancreatectomy of curative intent," Journal of Gastrointestinal Surgery, vol. 13, no. 2, pp. 349-353, 2009.

[12] K. J. Kelly, J. Wong, R. Gladdy et al., "Prognostic impact of RTPCR-based detection of peritoneal micrometastases in patients with pancreatic cancer undergoing curative resection," Annals of Surgical Oncology, vol. 16, no. 12, pp. 3333-3339, 2009.

[13] Y. Mataki, S. Takao, K. Maemura et al., "Carcinoembryonic antigen messenger RNA expression using nested reverse transcription-PCR in the peripheral blood during follow-up period of patients who underwent curative surgery for biliary pancreatic cancer: longitudinal analyses," Clinical Cancer Research, vol. 10, no. 11, pp. 3807-3814, 2004.

[14] G. Garcea, N. Ladwa, C. P. Neal, M. S. Metcalfe, A. R. Dennison, and D. P. Berry, "Preoperative neutrophil-to-lymphocyte ratio (NLR) is associated with reduced disease-free survival following curative resection of pancreatic adenocarcinoma," World Journal of Surgery, vol. 35, no. 4, pp. 868-872, 2011.

[15] M. Oshima, K. Okano, S. Muraki et al., "Immunohistochemically detected expression of 3 major genes (CDKN2A/p16, TP53, and SMAD4/DPC4) strongly predicts survival in patients with resectable pancreatic cancer," Annals of Surgery, vol. 258, no. 2, pp. 336-346, 2013.

[16] K. Nagai, R. Doi, F. Katagiri et al., "Prognostic value of metastin expression in human pancreatic cancer," Journal of Experimental and Clinical Cancer Research, vol. 28, no. 1, article 9, 2009.

[17] W. C. Foo, A. Rashid, H. Wung et al., "Loss of phosphatase and tensin homolog expression is associated with recurrence and poor prognosis in patients with pancreatic ductal adenocarcinoma," Human Pathology, vol. 44, no. 6, pp. 1024-1030, 2013.

[18] J. B. Bachet, R. Maréchal, P. Demetter et al., "Contribution of CXCR4 and SMAD4 in predicting disease progression pattern and benefit from adjuvant chemotherapy in resected pancreatic adenocarcinoma," Annals of Oncology, vol. 23, no. 9, pp. 23272335, 2012.

[19] M. Niedergethmann, B. Wostbrock, J. W. Sturm, F. Willeke, S. Post, and R. Hildenbrand, "Prognostic impact of cysteine proteases cathepsin B and cathepsin L in pancreatic adenocarcinoma," Pancreas, vol. 29, no. 3, pp. 204-211, 2004.

[20] M. Niedergethmann, R. Hildenbrand, B. Wostbrock et al., "High expression of vascular endothelial growth factor predicts early recurrence and poor prognosis after curative resection for ductal adenocarcinoma of the pancreas," Pancreas, vol. 25, no. 2, pp. 122-129, 2002.

[21] N. B. Jamieson, D. C. Morran, J. P. Morton et al., "MicroRNA molecular profiles associated with diagnosis, clinicopathologic criteria, and overall survival in patients with resectable pancreatic ductal adenocarcinoma," Clinical Cancer Research, vol. 18, no. 2, pp. 534-545, 2012.

[22] J. M. Winter, C. J. Yeo, and J. R. Brody, "Diagnostic, prognostic, and predictive biomarkers in pancreatic cancer," Journal of Surgical Oncology, vol. 107, no. 1, pp. 15-22, 2013.

[23] H. Koprowski, Z. Steplewski, K. Mitchell, M. Herlyn, D. Herlyn, and P. Fuhrer, "Colorectal carcinoma antigens detected by hybridoma antibodies," Somatic Cell Genetics, vol. 5, no. 6, pp. 957-971, 1979.

[24] M. Bloomston, T. S. Bekaii-Saab, K. Kosuri et al., "Preoperative carbohydrate antigen 19-9 is most predictive of malignancy in older jaundiced patients undergoing pancreatic resection," Pancreas, vol. 33, no. 3, pp. 246-249, 2006.

[25] P. Muscarella II, W. Fisher, J. A. Johnson, and W. S. Melvin, "Neoplastic disorders of the pancrease," in Clinical Pathology of Pancreatic Disorders, J. A. Lott, Ed., pp. 125-163, Humana Press, Totowa, NJ, USA, 1997.

[26] R. Lamerz, "Role of tumour markers, cytogenetics," Annals of Oncology, vol. 10, no. 4, pp. S145-S149, 1999.

[27] M. S. Woods, L. W. Traverso, R. A. Kozarek et al., "Characteristics of biliary tract complications during laparoscopic cholecystectomy: a multi-institutional study," The American Journal of Surgery, vol. 167, no. 1, pp. 27-34, 1994.

[28] D. Barone, M. Onetto, M. Conio et al., "CA 19-9 assay in patients with extrahepatic cholestatic jaundice," International Journal of Biological Markers, vol. 3, no. 2, pp. 95-100, 1988.

[29] K. Bhat, F. Wang, Q. Ma et al., "Advances in biomarker research for pancreatic cancer," Current Pharmaceutical Design, vol. 18, no. 17, pp. 2439-2451, 2012.

[30] S. R. Z. Abdel-Misih, I. Hatzaras, C. Schmidt et al., "Failure of normalization of CA19-9 following resection for pancreatic cancer is tantamount to metastatic disease," Annals of Surgical Oncology, vol. 18, no. 4, pp. 1116-1121, 2011.

[31] R. C. Montgomery, J. P. Hoffman, L. B. Riley, A. Rogatko, J. A. Ridge, and B. L. Eisenberg, "Prediction of recurrence and survival by post-resection CA 19-9 values in patients with adenocarcinoma of the pancreas," Annals of Surgical Oncology, vol. 4, no. 7, pp. 551-556, 1997.

[32] M. Carpelan-Holmström, J. Louhimo, U.-H. Stenman, H. Alfthan, and C. Haglund, "CEA, CA 19-9 and CA 72-4 improve the diagnostic accurary in gastrointestinal cancers," Anticancer Research, vol. 22, no. 4, pp. 2311-2316, 2002.

[33] G. Banfi, S. Bravi, A. Ardemagni, and A. Zerbi, "CA 19.9, CA 242 and CEA in the diagnosis and follow-up of pancreatic cancer," International Journal of Biological Markers, vol. 11, no. 2, pp. 7781, 1996.

[34] J. Glenn, W. M. Steinberg, S. H. Kurtzman, S. M. Steinberg, and W. F. Sindelar, "Evaluation of the utility of a radioimmunoassay for serum CA 19-9 levels in patients before and after treatment of carcinoma of the pancreas," Journal of Clinical Oncology, vol. 6, no. 3, pp. 462-468, 1988.

[35] M. Yasue, J. Sakamoto, S. Teramukai et al., "Prognostic values of preoperative and postoperative CEA and CA19.9 levels in pancreatic cancer," Pancreas, vol. 9, no. 6, pp. 735-740, 1994.

[36] K. Katsuragi, M. Yashiro, T. Sawada, H. Osaka, M. Ohira, and K. Hirakawa, "Prognostic impact of PCR-based identification of isolated tumour cells in the peritoneal lavage fluid of gastric cancer patients who underwent a curative R0 resection," The British Journal of Cancer, vol. 97, no. 4, pp. 550-556, 2007. 
[37] K. M. Dalal, Y. Woo, C. Galanis et al., "Detection of micrometastases in peritoneal washings of pancreatic cancer patients by the reverse transcriptase polymerase chain reaction," Journal of Gastrointestinal Surgery, vol. 11, no. 12, pp. 1598-1606, 2007.

[38] K. M. Dalal, Y. Woo, K. Kelly et al., "Detection of micrometastases in peritoneal washings of gastric cancer patients by the reverse transcriptase polymerase chain reaction," Gastric Cancer, vol. 11, no. 4, pp. 206-213, 2008.

[39] F. Miyazono, S. Takao, S. Natsugoe et al., "Molecular detection of circulating cancer cells during surgery in patients with biliarypancreatic cancer," The American Journal of Surgery, vol. 177, no. 6, pp. 475-479, 1999.

[40] K. Uchikura, S. Takao, A. Nakajo et al., "Intraoperative molecular detection of circulating tumor cells by reverse transcriptionpolymerase chain reaction in patients with biliary-pancreatic cancer is associated with hematogenous metastasis," Annals of Surgical Oncology, vol. 9, no. 4, pp. 364-370, 2002.

[41] K. Tjensvoll, O. Nordgård, and R. Smaaland, "Circulating tumor cells in pancreatic cancer patients: methods of detection and clinical implications," International Journal of Cancer, vol. 134, no. 1, pp. 1-8, 2014.

[42] D. C. McMillan, "Systemic inflammation, nutritional status and survival in patients with cancer," Current Opinion in Clinical Nutrition and Metabolic Care, vol. 12, no. 3, pp. 223-226, 2009.

[43] C. S. D. Roxburgh and D. C. McMillan, "Role of systemic inflammatory response in predicting survival in patients with primary operable cancer," Future Oncology, vol. 6, no. 1, pp. 149$163,2010$.

[44] I. Bhatti, O. Peacock, G. Lloyd, M. Larvin, and R. I. Hall, "Preoperative hematologic markers as independent predictors of prognosis in resected pancreatic ductal adenocarcinoma: neutrophil-lymphocyte versus platelet-lymphocyte ratio," The American Journal of Surgery, vol. 200, no. 2, pp. 197-203, 2010.

[45] M. Aliustaoglu, A. Bilici, M. Seker et al., "The association of pretreatment peripheral blood markers with survival in patients with pancreatic cancer," Hepato-Gastroenterology, vol. 57, no. 99-100, pp. 640-645, 2010.

[46] X. An, P.-R. Ding, Y.-H. Li et al., "Elevated neutrophil to lymphocyte ratio predicts survival in advanced pancreatic cancer," Biomarkers, vol. 15, no. 6, pp. 516-522, 2010.

[47] C. H. Crane, G. R. Varadhachary, J. S. Yordy et al., "Phase II trial of cetuximab, gemcitabine, and oxaliplatin followed by chemoradiation with cetuximab for locally advanced (T4) pancreatic adenocarcinoma: correlation of Smad4(Dpc4) immunostaining with pattern of disease progression," Journal of Clinical Oncology, vol. 29, no. 22, pp. 3037-3043, 2011.

[48] M. Tascilar, H. G. Skinner, C. Rosty et al., "The SMAD4 protein and prognosis of pancreatic ductal adenocarcinoma," Clinical Cancer Research, vol. 7, no. 12, pp. 4115-4121, 2001.

[49] C. A. Lacobuzio-Donahue, B. Fu, S. Yachida et al., "DPC4 gene status of the primary carcinoma correlates with patterns of failure in patients with pancreatic cancer," Journal of Clinical Oncology, vol. 27, no. 11, pp. 1806-1813, 2009.

[50] J. M. Winter, L. H. Tang, D. S. Klimstra et al., "Failure patterns in resected pancreas adenocarcinoma: lack of predicted benefit to SMAD4 expression," Annals of Surgery, vol. 258, no. 2, pp. 331-335, 2013.

[51] T. Ohtaki, Y. Shintani, S. Honda et al., "Metastasis suppressor gene KiSS-1 encodes peptide ligand of a G-protein-coupled receptor," Nature, vol. 411, no. 6837, pp. 613-617, 2001.

[52] M. Kotani, M. Detheux, A. Vandenbogaerde et al., "The metastasis suppressor gene KiSS-1 encodes kisspeptins, the natural ligands of the orphan G protein-coupled receptor GPR54," Journal of Biological Chemistry, vol. 276, no. 37, pp. 34631-34636, 2001.

[53] T. Masui, R. Doi, T. Mori et al., "Metastin and its variant forms suppress migration of pancreatic cancer cells," Biochemical and Biophysical Research Communications, vol. 315, no. 1, pp. 85-92, 2004.

[54] H. Ying, K. G. Elpek, A. Vinjamoori et al., "PTEN is a major tumor suppressor in pancreatic ductal adenocarcinoma and regulates an NF- $\kappa \mathrm{B}$-cytokine network," Cancer Discovery, vol. 1, no. 2, pp. 158-169, 2011.

[55] R. Hill, J. H. Calvopina, C. Kim et al., "PTEN loss accelerates KrasG12D-induced pancreatic cancer development," Cancer Research, vol. 70, no. 18, pp. 7114-7124, 2010.

[56] S. Shroff, M. J. Overman, A. Rashid et al., "The expression of PTEN is associated with improved prognosis in patients with ampullary adenocarcinoma after pancreaticoduodenectomy," Archives of Pathology \& Laboratory Medicine, vol. 137, no. 11, pp. 1619-1626, 2013.

[57] R. Maréchal, P. Demetter, N. Nagy et al., "High expression of CXCR4 may predict poor survival in resected pancreatic adenocarcinoma," The British Journal of Cancer, vol. 100, no. 9, pp. 1444-1451, 2009.

[58] V. Ellenrieder, G. Adler, and T. M. Gress, "Invasion and metastasis in pancreatic cancer," Annals of Oncology, vol. 10, supplement 4, pp. S46-S50, 1999.

[59] M. J. Duffy, "The role of proteolytic enzymes in cancer invasion and metastasis," Clinical and Experimental Metastasis, vol. 10, no. 3, pp. 145-155, 1992.

[60] R. Kuliawat, J. Klumperman, T. Ludwig, and P. Arvan, "Differential sorting of lysosomal enzymes out of the regulated secretory pathway in pancreatic $\beta$-cells," Journal of Cell Biology, vol. 137, no. 3, pp. 595-608, 1997.

[61] F. M. Tumminello, G. Leto, G. Pizzolanti et al., "Cathepsin D, $\mathrm{B}$ and $\mathrm{L}$ circulating levels as prognostic markers of malignant progression," Anticancer Research, vol. 16, no. 4, pp. 2315-2319, 1996.

[62] J. Folkman, K. Watson, D. Ingber, and D. Hanahan, "Induction of angiogenesis during the transition from hyperplasia to neoplasia," Nature, vol. 339, no. 6219, pp. 58-61, 1989.

[63] N. Weidner, J. P. Semple, W. R. Welch, and J. Folkman, “Tumor angiogenesis and metastasis-correlation in invasive breast carcinoma," New England Journal of Medicine, vol. 324, no. 1, pp. 1-8, 1991.

[64] J. Itakura, T. Ishiwata, H. Friess et al., "Enhanced expression of vascular endothelial growth factor in human pancreatic cancer correlates with local disease progression," Clinical Cancer Research, vol. 3, no. 8, pp. 1309-1316, 1997.

[65] M. Niedergethmann, R. Hildenbrand, G. Wolf, C. S. Verbeke, A. Richter, and S. Post, "Angiogenesis and cathepsin expression are prognostic factors in pancreatic adenocarcinoma after curative resection," International Journal of Pancreatology, vol. 28, no. 1, pp. 31-39, 2000.

[66] Y. Seo, H. Baba, T. Fukuda, M. Takashima, and K. Sugimachi, "High expression of vascular endothelial growth factor is associated with liver metastasis and a poor prognosis for patients with ductal pancreatic adenocarcinoma," Cancer, vol. 88, no. 10, pp. 2239-2245, 2000.

[67] H. A. Arafat, Q. Gong, G. Chipitsyna, A. Rizvi, C. T. Saa, and C. J. Yeo, "Antihypertensives as novel antineoplastics: angiotensin-I-converting enzyme inhibitors and angiotensin II 
type 1 receptor blockers in pancreatic ductal adenocarcinoma," Journal of the American College of Surgeons, vol. 204, no. 5, pp. 996-1005, 2007.

[68] T. Sharma, R. Dhingra, S. Singh et al., "Aflibercept: a novel VEGF targeted agent to explore the future perspectives of antiangiogenic therapy for the treatment of multiple tumors," MiniReviews in Medicinal Chemistry, vol. 13, no. 4, pp. 530-540, 2013.

[69] A. Gaya and V. Tse, "A preclinical and clinical review of aflibercept for the management of cancer," Cancer Treatment Reviews, vol. 38, no. 5, pp. 484-493, 2012.

[70] J. Rodríguez-Pascual, P. Sha, E. García-García et al., "A preclinical and clinical study of mycophenolate mofetil in pancreatic cancer," Investigational New Drugs, vol. 31, no. 1, pp. 14-19, 2013.

[71] M. Bloomston, W. L. Frankel, F. Petrocca et al., "MicroRNA expression patterns to differentiate pancreatic adenocarcinoma from normal pancreas and chronic pancreatitis," Journal of the American Medical Association, vol. 297, no. 17, pp. 1901-1908, 2007.

[72] C. W. Steele, K. A. Oien, C. J. McKay, and N. B. Jamieson, "Clinical potential of microRNAs in pancreatic ductal adenocarcinoma," Pancreas, vol. 40, no. 8, pp. 1165-1171, 2011.

[73] B. E. Kadera, L. Li, P. A. Toste et al., "MicroRNA-21 in pancreatic ductal adenocarcinoma tumor-associated fibroblasts promotes metastasis," PLoS ONE, vol. 8, no. 8, Article ID e71978, 2013.

[74] Z. Zhu, Y. Xu, J. Du, J. Tan, and H. Jiao, "Expression of microRNA-218 in human pancreatic ductal adenocarcinoma and its correlation with tumor progression and patient survival," Journal of Surgical Oncology, vol. 109, no. 2, pp. 89-94, 2014. 


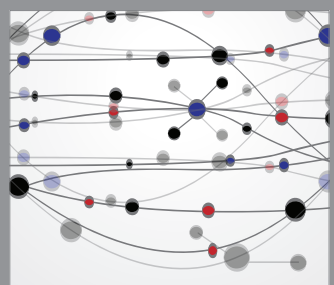

The Scientific World Journal
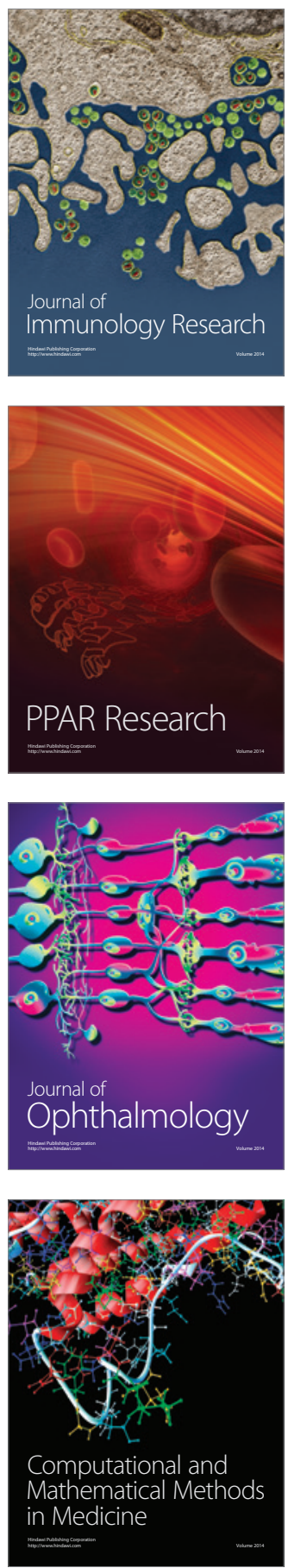

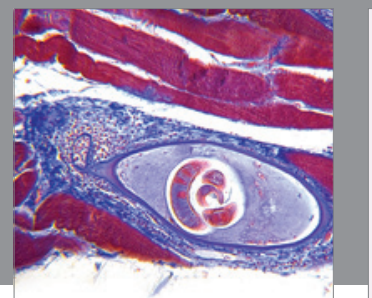

Gastroenterology

Research and Practice
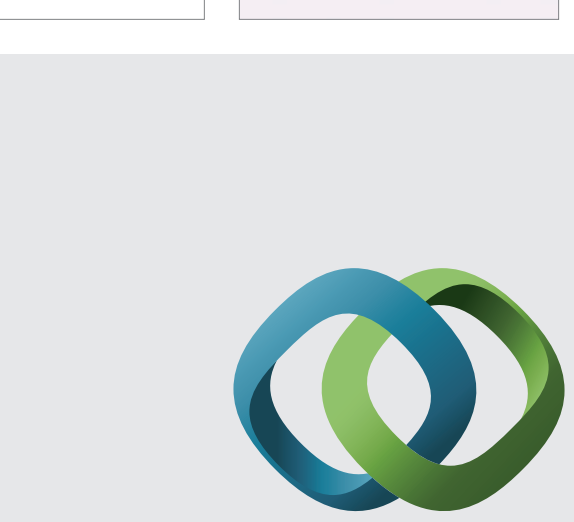

\section{Hindawi}

Submit your manuscripts at

http://www.hindawi.com
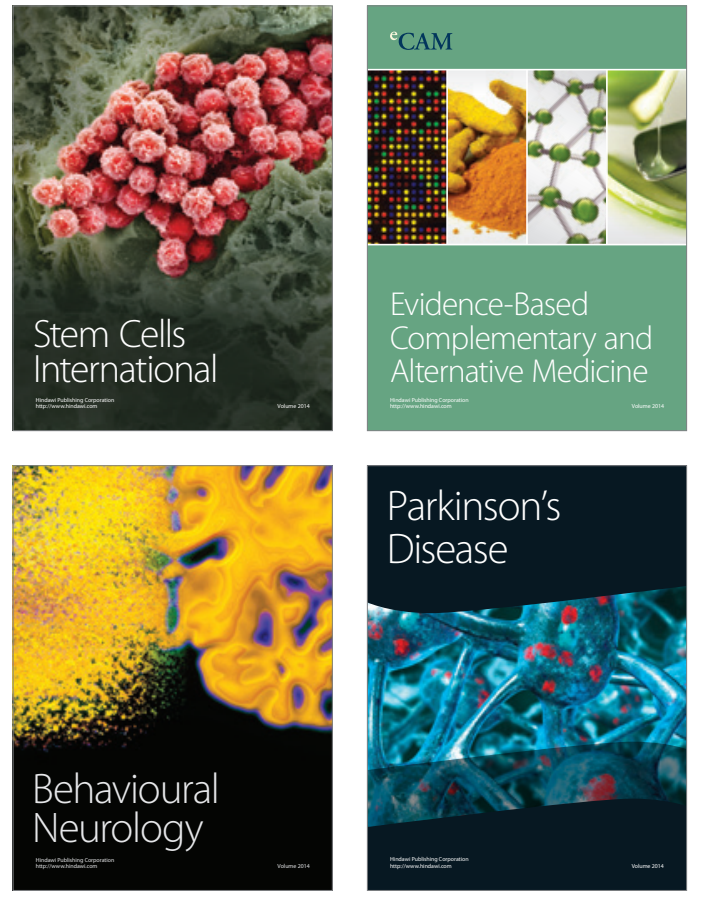
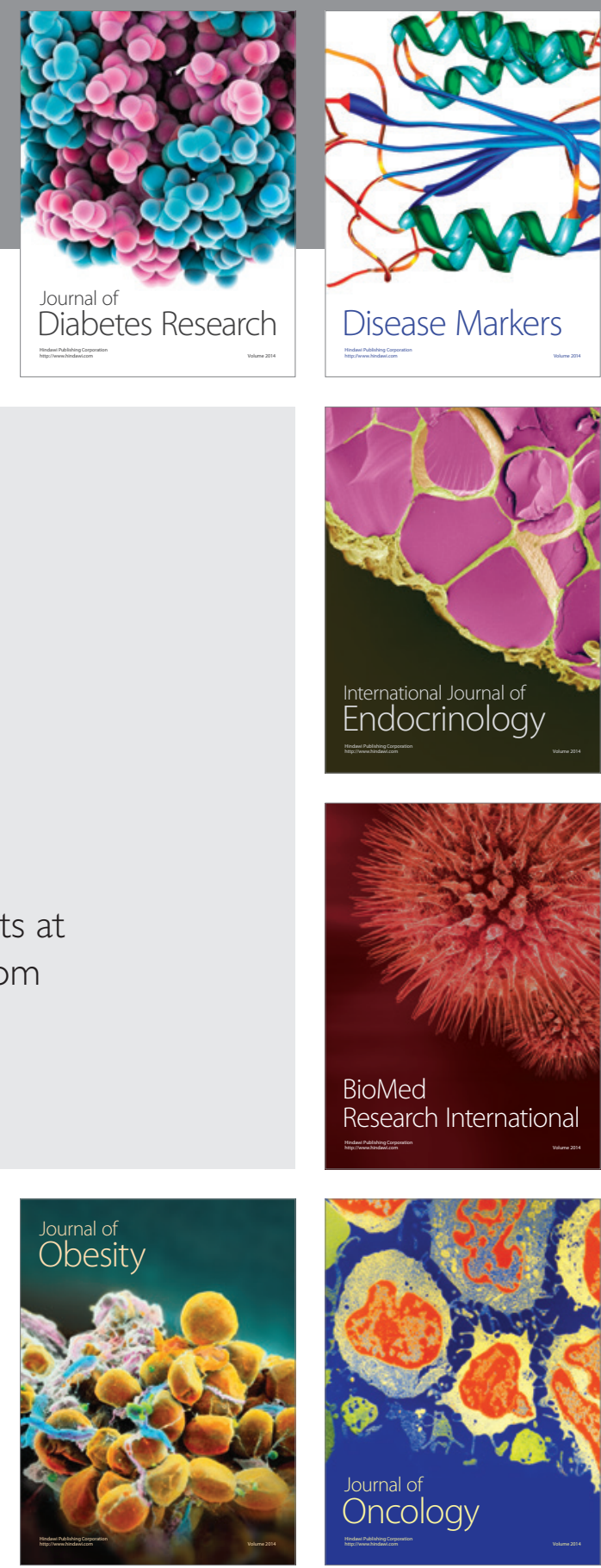

Disease Markers
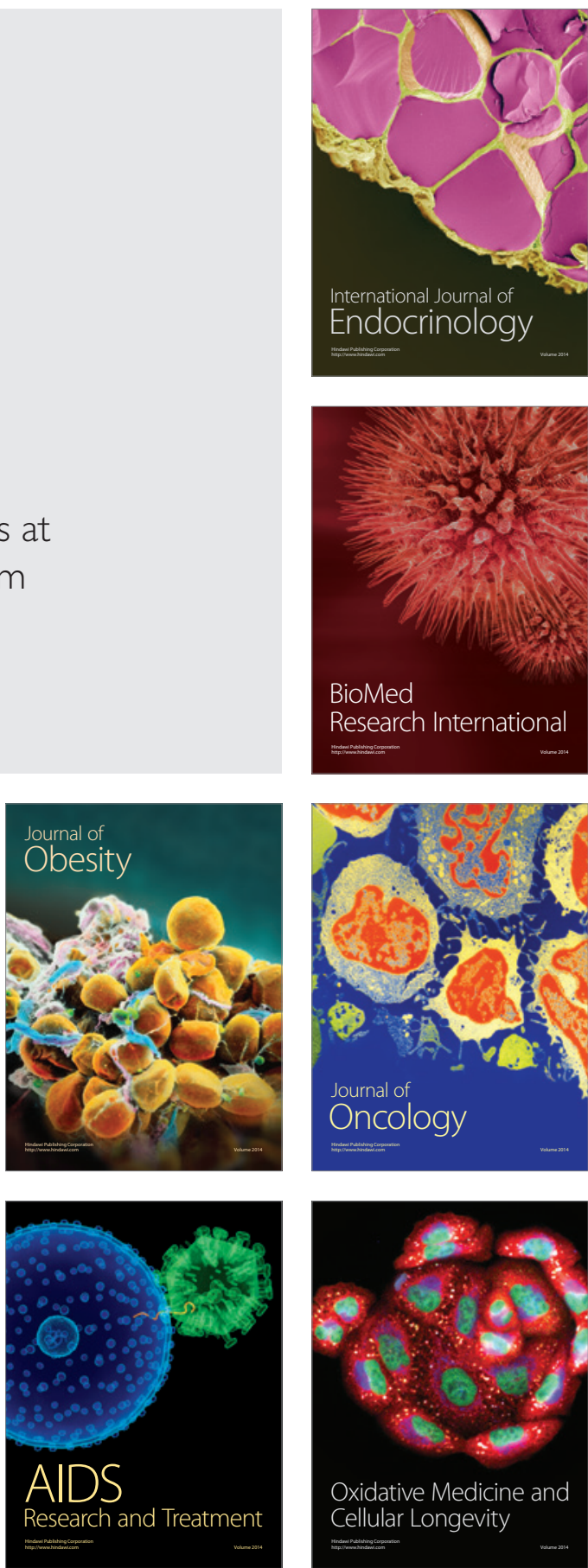